\title{
Collision Vehicle Detection System Based on GPS/INS Integration
}

\author{
Neda Navidi, Rene Jr. Landry \\ LASSENA Laboratory, Ecole de Technologie Superieure, Montreal, Canada \\ Email: Neda.navidi@lassena.etsmtl.ca
}

How to cite this paper: Navidi, N. and Landry, R.Jr. (2017) Collision Vehicle Detection System Based on GPS/INS Integration. Journal of Computer and Communications, 5, 48-70. https://doi.org/10.4236/jcc.2017.52006

Received: December 22, 2016

Accepted: February 25, 2017

Published: February 28, 2017

Copyright $\odot 2017$ by authors and Scientific Research Publishing Inc. This work is licensed under the Creative Commons Attribution International License (CC BY 4.0).

http://creativecommons.org/licenses/by/4.0/

(c) (i) Open Access

\begin{abstract}
Continuous vehicle tracking as well as detecting accidents, are significant services that are needed by many industries including insurance and vehicle rental companies. The main goal of this paper is to provide methods to detect the position of car accident. The models consider GPS/INS-based navigation algorithm, calibration of navigational sensors, a de-nosing method as long as vehicle accident, expressed by a set of raw measurements which are obtained from various environmental sensors. In addition, the location-based accident detection model is tested in different scenarios. The results illustrate that under harsh environments with no GPS signal, location of accident can be detected. Also results confirm that calibration of sensors has an important role in position correction algorithm. Finally, the results present that the proposed accident detection algorithm can recognize accidents and related its positions.
\end{abstract}

\section{Keywords}

Accident Detection, GPS, INS, EKF, GPS/INS Integration, Calibration, De-Noising

\section{Introduction}

In recent years, the rate of vehicle accident fatalities has been one of the main concerns in rural and urban communities. Public Health Agency of Canada (PHAC), has reported more than 2.209 fatalities and 11.451 serious injuries in every year [1]. Car accidents may impose expenses to governments, namely as needed medical treatments, rehabilitation assistance, and property damages. Such expenses are estimated to be more than one hundred billion dollars per year in Canada [1].

Vehicle tracking is one of the significant concerns for the insurance and the vehicle rental companies. Monitoring driver's behavior helps develop the pricing 
solutions based on car usage (PAYD: Pay As You Drive), the driving habits (PHYD: Pay How You Drive) or the area of operation (PWYD: Pay Where You Drive) [2].

Accuracy requirement of location based services mostly depends on Global Positioning System (GPS) and global navigation satellite systems (GNSS) to obtain an admitted navigational solution. GPS and GNSS receivers require perfect operating conditions for providing a valid solution. However, these conditions cannot be realized while driving in harsh environments such urban canyons or under dense foliage. Whereas, GNSS and GPS operate affected by Line of Sight (LOS) signal propagation, harsh environments reduce the maximum efficiency of these devices. So we cannot count on them as the all-environment positioning technologies [3].

Advanced commercial vehicle services-such as car insurance companies need to vanquish the motioned problems of GPS and GNSS to provide higher customer supports. An encouraging solution to achieve a high-precision navigation system, is to fuse the GPS data with low-cost Micro-Electro-Mechanical Systems (MEMS)-based Inertial Measurement Units (IMUs) which are self-governing navigation devices. In fact, an INS/GPS integrated system can provide a state-ofthe-art land navigation system with a superior achievement in comparison with GPS or INS stand-alone. The conventional Extended Kalman Filter (EKF) extensively has been employed to the integration of INS and GPS which can determine the optimal estimation of the system state vector with minimum mean and square errors [4] [5].

EKF needs to perform under the appropriate defined errors and dynamic models in its initialization process, which requires the consideration of the noise modeling. Therefore, to provide the good performance, EKF requires the good definition on the dynamic process of the system and measurement model based on Gaussian white noises. This paper has designed an EKF-based GPS/INS to have a full coverage in GPS outages.

In EKF-based GPS/INS integration, IMUs can estimate the position and the attitude of the vehicle by employing an Inertial Navigation System (INS) mechanization process (accelerometers and gyroscopes), which is an integration of linear accelerations and angular rates. However, low-cost INSs perform accurately just for a limited time as their positioning and attitudinal errors increase steadily with time. In fact, the inertial sensor errors are generally composed of two components: systematic errors and stochastic errors [6] [7]. These errors, if not dealt with, will have a significant negative impact on the quality of the navigation solution and the accuracy of vehicular tracking output.

This paper has developed calibration techniques for reducing low-cost sensors systematic errors using a Powell's Dog Leg Method. The raw data of IMUs have to be exploited in stationary condition, when it is processed with the Powell's Dog Leg as an iterative method for performing the calibration procedures [8] [9]. Considering the stochastic errors, as these errors have High Frequency (HF) term, it is needed to decrease HF term in order to develop the accuracy of the 
low-cost inertial sensors [10]. Wavelet De-noising Technique (WDT) has been utilized in this paper, due to its significant role in removing the HF noises.

Accident detection technologies are the technologies to trigger and activate Supplemental Restraint System (SRS) like airbags and seatbelts used in different vehicles [11]. This paper proposed to use acceleration and curvature of the moving vehicle as two significant parameters which are obtained by calculation of accelerometers and gyroscopes measurement. As soon as an accident is detected by the accident detection algorithm, the navigation system can reveal the position of the accident.

The paper is organized as following: Section 2 presents the background of the work. Section 3 gives the proposed methodology for the accident detection process. Section 4 presents the experimental results and their analysis in various scenarios. Section 5 presents the conclusions of this paper.

\section{Background}

This section presents details on background of techniques and ideas used in this study. More specifically, four main algorithms will be considered in this study: 1) calibration of systematic error of accelerometers and gyroscopes, 2) removing the part of stochastic error in the mentioned sensors by de-nosing techniques, 3) integration of GPS and INS to have a full coverage for navigation system, and 4) detection of accident based on dynamic of the moving vehicle. Algorithms (1) (3) need more background and details which are presented in this section.

\subsection{Calibration of Systematic Errors}

The systematic errors relate to the calibration parameters comprising the scale factor, misalignment, and bias errors of the sensors. The calibration procedure can characterize and then delete the systematic errors from raw measurements [12] [13] [14]. To remove the systematic error, it is necessary to compare the raw data of IMU with a known reference and then determine the systematic errors which agree with that reference. The accelerometer and gyroscope outputs can be written as:

$$
\tilde{X}^{i}=S F^{i} \times M^{i} \times X^{i}+B^{i}+w^{i}
$$

where, $\tilde{X}^{i}, X^{i}, w^{i}$, are the sensor outputs, the sensor real data, and the noise term, respectively. $S F^{i}, M^{i}$, and $B^{i}$ are respectively the scale factor, the misalignment and the bias errors of the sensors, which are called the calibration parameters (See Figure 1). In this paper, “ $P$ ” index shows the gyroscope with " $g$ " and accelerometers with " $a$ ". $B^{i}, S F^{i}$ and $M^{i}$ can be defined as follows:

$$
\begin{gathered}
B^{i}=\left[\begin{array}{lll}
B_{x}^{i} & B_{y}^{i} & B_{z}^{i}
\end{array}\right]^{\mathrm{T}} \\
S F^{i}=\operatorname{diag}\left[\begin{array}{ccc}
S F_{x}^{i} & S F_{y}^{i} & S F_{z}^{i}
\end{array}\right] \\
M^{i}=\left[\begin{array}{ccc}
1 & -M_{z}^{i} & +M_{y}^{i} \\
0 & 1 & -M_{x}^{i} \\
0 & 0 & 1
\end{array}\right]
\end{gathered}
$$


where, lower index $(x, y$ and $z$ ) shows the calibration parameters related to $x, y$ and $z$ axes.

\subsection{Wavelet Denoising Technique (WDT)}

Wavelet De-noising Technique (WDT) is based on the wavelet filtering method, which can eliminate the stochastic error in the measurements of the inertial sensors at High Frequencies (HFs) without altering important information contained in the signal [15] [16]. A Multi-Resolution Analysis (MRA) algorithm was performed on the WDT, which can decompose a signal into different sub-bands with various time and frequency resolutions.

Conceptually, in WDT, signal $x(n)$ is filtered by one high-pass filter and one low-pass filter with down-sampling by two $(\downarrow 2)$ in the continuous Level of Decompositions (LODs), as shown in Figure 2. The maximum amount of information about the original signal $x(n)$ was contained in the approximation coefficients $\left(A^{k}\right)$ in each sub-band. Hence, the minority information of $x(n)$ was contained in HF noise components, which were identified by detailed coefficients $\left(D^{k}\right)$.

\subsection{GPS/INS Integration Process}

Integration of INSs and GPSs through loosely coupled structure, not only maintains independency and redundancy of stand-alone GPS and INS solutions; but

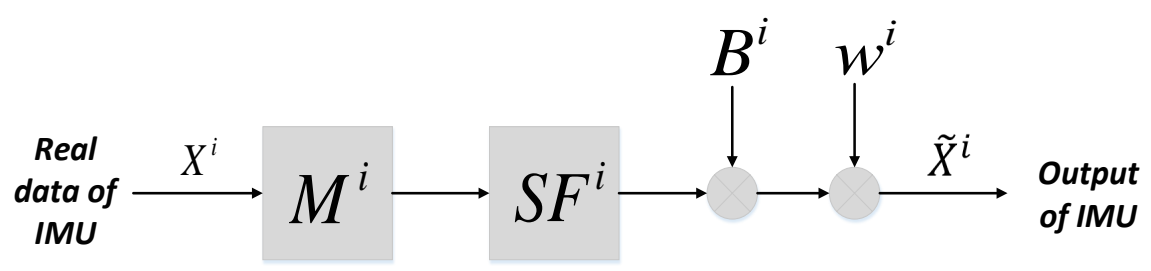

Figure 1. Modelled systematic errors in IMU.

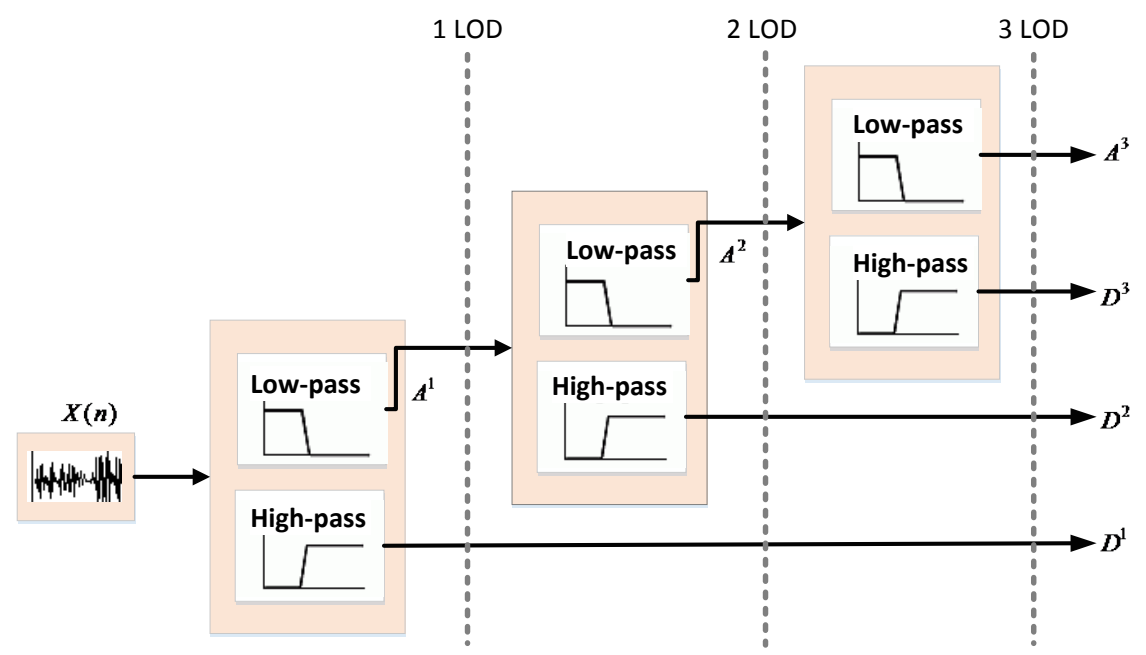

Figure 2. Application of Wavelet De-noising Technique (WDT) with different Level of Decompositions (LODs). 
also, it can provide more robustness navigation solution [17]. Loosely coupled structure presents a closed-loop architecture that allows the correction of certain errors of the INS system. This structure is generally preferable in the INS/GPS integration as being composed of three distinct entities, which are the standalone GPS solution, the stand-alone INS solution and the INS/GPS coupled solution. This architecture, as presented in Figure 3, is shared by several authors [18] [19] [20]. In the architecture, a filter is used to couple the GPS and INS solutions (position and velocity) to estimate some system error's parameters. The estimated parameters correspond to the errors of position, velocity, and attitude of INS solution.

The filters in the Figure 3, are EKFs; which are recognized as the most conventional method to estimate the navigation systems. The derivation of the error model to be applied in the EKF starts with the construction of the full scale true error models, whose order is decided on the complexity of the problem [21]. The error model of the dynamical model includes three errors of position, three errors of velocity, and three errors of attitude, augmented by some sensor error. INS/GPS integration reduces only the long-term errors with this error model. Thus, the remaining error budget is mainly affected by short-term error sources [22]. A block diagram of EKF is shown in Figure 4.

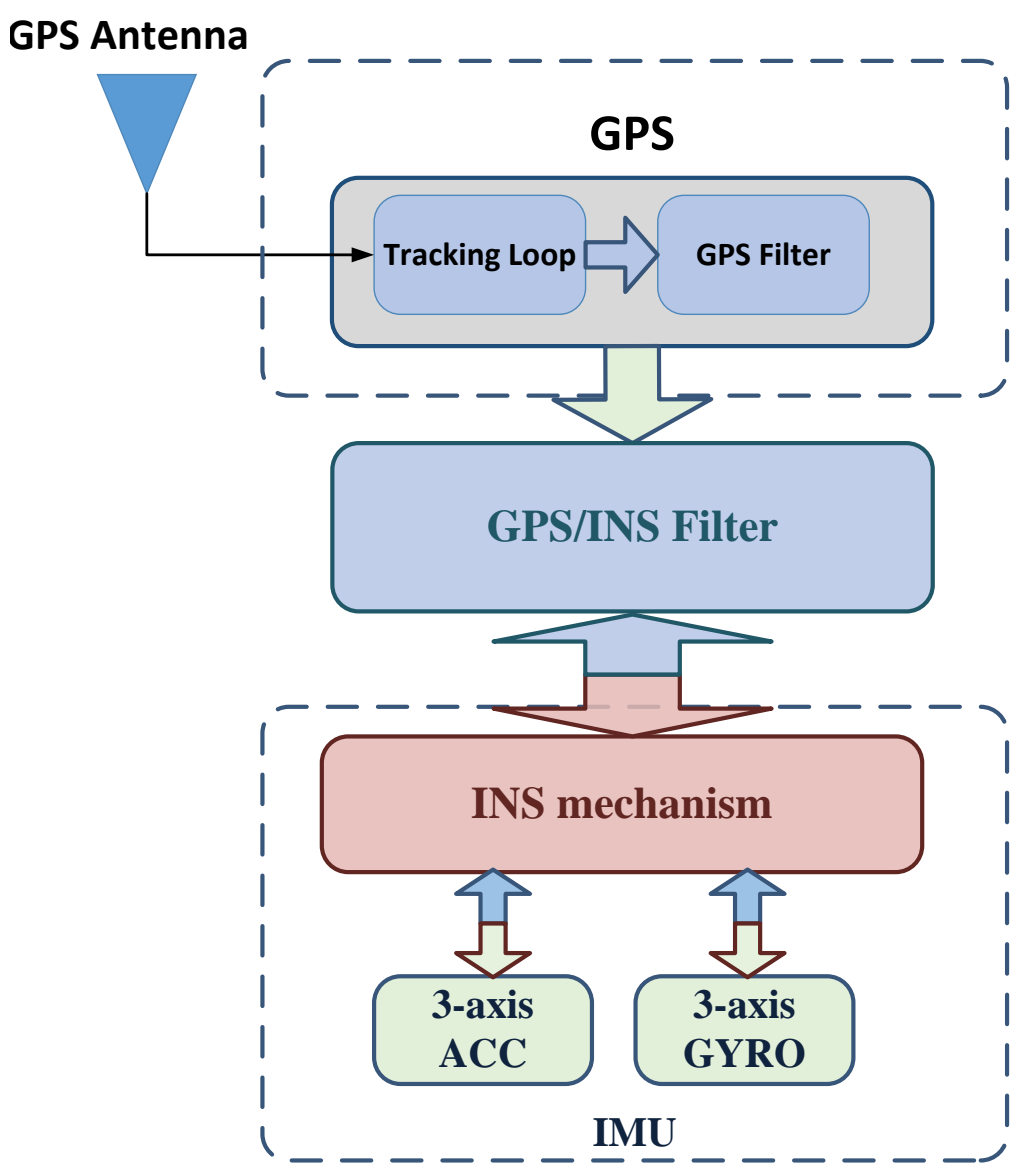

Figure 3. Loosely coupled INS/GPS integration. 


\section{Proposed Methods}

This paper proposes to combine four independent and complementary solutions into a global accident detection system to provide stable and accurate positioning of car accident even in severe urban environments. The proposed solutionsconsist of compensating the systematic errors of low-cost inertial using Powell's Dog Leg algorithm; augmenting the navigation solution with combined GPS and INS using EKF; and exploiting the inertial sensor to estimate the dynamics of vehicle to extract the accident. Figure 5 shows that how four solutions are combined in this study. This section presents details on these proposed solutions.

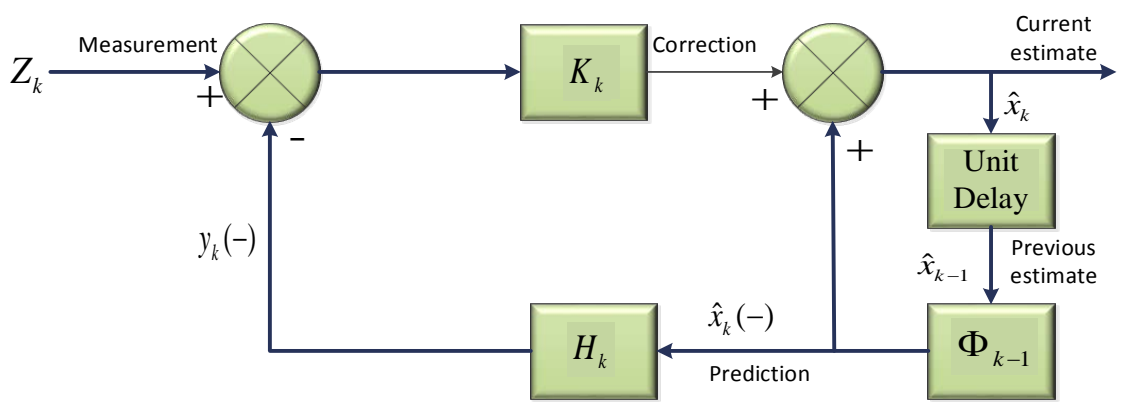

Figure 4. A block diagram of Extended Kalman Filter.

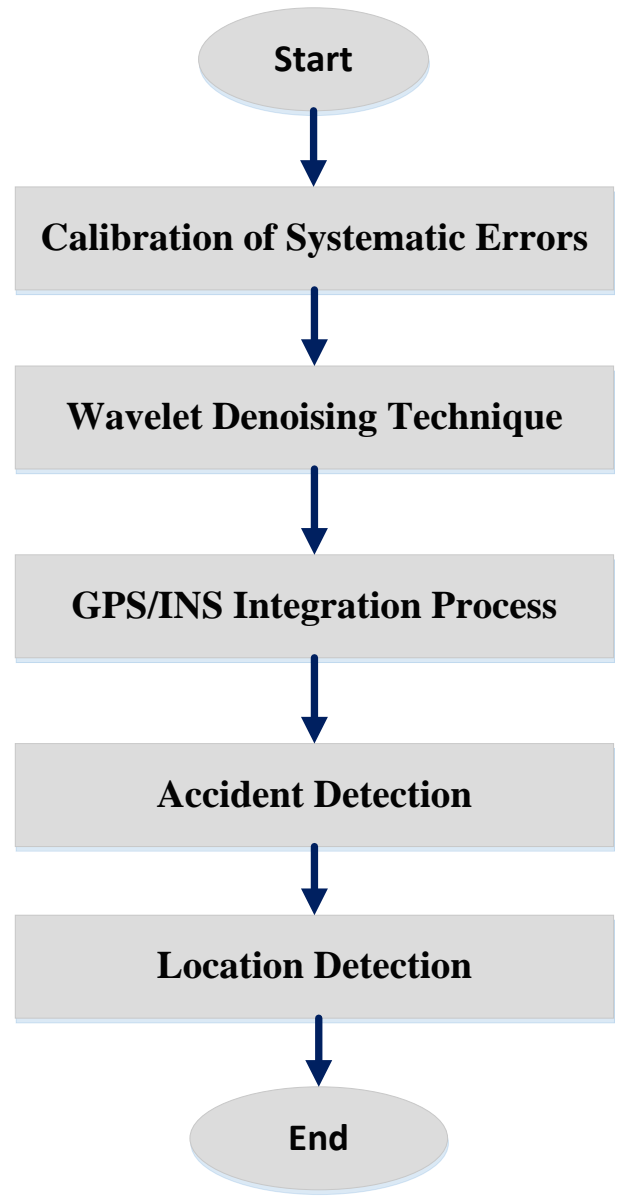

Figure 5. Flowchart of the auto-accident detection. 


\subsection{Powell's Dog Leg Calibration Method}

According to [23]-[29], it is possible to estimate the sensor real data $\left(X^{i}\right)$ which is presented in section 2.2.1, by Equations (5) and (6):

$$
\begin{gathered}
X^{i}=f\left(\tilde{X}^{i}, \varsigma\right)=M^{i} S F^{i-1}\left(\tilde{X}^{i}-B^{i}\right) \\
\varsigma=\left[\begin{array}{lllllllll}
S F_{x}^{i} & S F_{y}^{i} & S F_{z}^{i} & M_{x}^{i} & M_{y}^{i} & M_{z}^{i} & B_{x}^{i} & B_{y}^{i} & B_{z}^{i}
\end{array}\right]
\end{gathered}
$$

It is expected that the measured data is the same as the real data. Then the objective function should be defined to minimize the square error between these values; so, it can be expressed by:

$$
\hat{\zeta}=\sum_{k=0}^{A B-1}\left[\left\|X^{i}\right\|^{2}-\left\|f\left(\tilde{X}^{i}, \varsigma\right)\right\|^{2}\right]^{2}=0
$$

where, $A$ and $B$ are the numbers of the position and the sample of the recorded data, respectively. So, $\tilde{X}^{a}$ was assumed as the earth's gravity vector for calibration of the accelerometers and $\tilde{X}^{g}$ as the earth rotation rate vector for calibration of the gyroscopes.

This paper proposes a simple calibration procedure, based on Powell's Dog Leg Method to enhance the performance of the calibration procedure. The Powell's Dog Leg algorithm is an iterative optimization algorithm allowing to solve quickly some problems related to the minimization of an objective function, notably the nonlinear least squares problems as it is for the calibration of the inertial sensors. This algorithm, which is similar to the Guass-Newton and gradient descent algorithms, consists in iteratively computing a perturbation to be added to the previous state vector in order to reduce the cost function. The Powell's Dog Legis summarized by the pseudo-code illustrated in Figure 6.

\subsection{GPS/INS EKF-Based Integration}

INS mechanization equations based on the mathematical process show the navigational information from the inertial sensor measurements. The model of the motion of the moving vehicle in this paper is presented by Equation (8) [30] [31]:

$$
\left[\begin{array}{l}
\frac{\dot{\boldsymbol{R}}_{b}^{n}}{\dot{\dot{V}}^{n}} \\
\delta \dot{\dot{P}}^{n}
\end{array}\right]=\left[\begin{array}{l}
\boldsymbol{R}_{b}^{n}\left(\underline{\Omega}_{i b}^{b}-\Omega_{i l}^{l}\right) \\
\underline{f}^{n}+\underline{g}^{n}-\left(\underline{\Omega}_{e n}^{n}+2 \underline{\Omega}_{i e}^{n}\right) \times \underline{V}^{n} \\
T \cdot \underline{V}^{n}
\end{array}\right]
$$

The attitude of the moving body inside the INS algorithm is obtained by integrating the propagation equation of direction cosines matrix (DCM) which is shown with $R_{b}^{n}$. The rotational rate of the frame relative to the terrestrial inertial frame $\left(\underline{\Omega}_{i b}^{b}\right)$ is measured directly by the gyroscopes, while the rotational rate of the local reference with respect to the terrestrial inertial frame $\left(\Omega_{i l}^{l}\right)$ must be calculated from the rotational rate of the Earth, the $R_{b}^{n}$ can be ob tained by $R_{b}^{l} * R_{l}^{n}$. In the mentioned equation, $R_{l}^{n}=R_{n}^{l}=\left[\begin{array}{ccc}0 & 1 & 0 \\ 1 & 0 & 0 \\ 0 & 0 & -1\end{array}\right]$ and $R_{b}^{l}$ 
can be calculated by Equation (9) [32]:

$$
R_{b}^{l}=\left[\begin{array}{ccc}
\cos \theta \cos \psi & -\cos \phi \sin \psi+\sin \phi \sin \theta \cos \psi & \sin \phi \sin \psi+\cos \phi \sin \theta \cos \psi \\
\cos \theta \sin \psi & \cos \phi \cos \psi+\sin \phi \sin \theta \sin \psi & -\sin \phi \cos \psi+\cos \phi \sin \theta \sin \psi \\
-\sin \theta & \sin \phi \cos \theta & \cos \phi \cos \theta
\end{array}\right]
$$

Euler angles can be defined as the functions of the matrix $R_{b}^{l}$ as the following:

$$
\begin{gathered}
\psi=\tan ^{-1} \frac{R_{b 21}^{l}}{R_{b 11}^{l}} \\
\phi=\tan ^{-1} \frac{R_{b 32}^{l}}{R_{b 33}^{l}} \\
\theta=\tan ^{-1} \frac{-R_{b 31}^{l}}{\sqrt{R_{b 32}^{l 2}+R_{b 33}^{l}{ }^{2}}}
\end{gathered}
$$

$$
\begin{aligned}
& \text { Start } \\
& i=0 ; x=x_{0} ; \sigma=\sigma_{0} ; s=J(x)^{\mathrm{T}} f(x) \\
& \text { find }=\left(\|f(x)\|_{\infty}<\varepsilon_{3}\right) \text { or }\left(\|s\|_{\infty}<\varepsilon_{1}\right) \\
& \text { While (not find) and } i<i_{\max } \\
& i=i+1 ; \quad \alpha=-\left(h_{s d}^{\mathrm{T}} J(x)^{\mathrm{T}} f(x)\right) /\left(\left\|f(x) h_{s d}\right\|^{2}\right) \\
& h_{\text {sd }}=-\alpha s \text { solve } J(x) h_{g n} \cong-f(x) \\
& \text { Computer } h_{d l}: h_{d l}=\alpha h_{s d}+\beta\left(h_{g n}-\alpha h_{s d}\right) \\
& \beta=\left(\sigma^{2}-\|a\|^{2}\right) /\left(c+\sqrt{c^{2}+\|b-a\|^{2}\left(\sigma^{2}-\|a\|^{2}\right)}\right) \\
& \text { if }\left\|h_{d l}\right\|<\varepsilon_{2}\left(\|x\|+\varepsilon_{2}\right) \\
& \text { find }=\text { True } \\
& \text { else } \\
& x_{\text {new }}=x+h_{d l} \\
& \zeta=\left(F(x)-F\left(x_{\text {new }}\right)\right) /\left(L(0)-L\left(h_{d l}\right)\right) \\
& \text { if } \zeta>0 \\
& x=x_{\text {new }} ; s=J(x)^{\mathrm{T}} f(x) \\
& \text { find }=\left(\|f(x)\|_{\infty}<\varepsilon_{3}\right) \text { or }\left(\|S\|_{\infty}<\varepsilon_{1}\right) \\
& \text { if } \zeta>3 / 4 \\
& \sigma=\max \left\{\sigma, 3 *\left\|h_{d l}\right\|\right\} \\
& \text { if } \zeta<1 / 4 \\
& \sigma=\sigma / 2 ; \quad \text { find }=\sigma<\varepsilon_{2}\left(\|x\|+\varepsilon_{2}\right)
\end{aligned}
$$

Figure 6. Pseudo-code of Powell's Dog Leg Method. 
where $\psi, \theta$ and $\phi$ are the components of Euler angle, namely, Azimuth, roll and pitch, respectively. The initial Euler angle based on initial alignment can be obtained by Equations (13)-(17):

$$
\begin{gathered}
\theta=\sin ^{-1} \frac{a c c_{x}}{g} \\
\phi=\tan ^{-1} \frac{a c c_{y}}{a c c_{z}}
\end{gathered}
$$

where, $a c c_{x}$ and $a c c_{z}$ are the magnetometer measurements in $x$ and $z$ axes, respectively. $\psi$ can be calculated by:

$$
\psi=-\tan ^{-1} \frac{M_{y}}{M_{x}}
$$

where, $M_{x}$ and $M_{y}$ can be calculated by magnetometer measurements by Equations (16) and (17):

$$
\begin{gathered}
M_{x}=m a g_{x} \cos \theta+m a g_{y} \sin \phi \sin \theta+m a g_{z} \cos \phi \sin \theta \\
M_{y}=\operatorname{mag}_{y} \cos \phi-m a g_{z} \sin \phi
\end{gathered}
$$

where, $\operatorname{mag}_{x}, \operatorname{mag}_{y}$ and $\mathrm{mag}_{z}$ are the magnetometer measurements in $x, y$ and $z$ axes, respectively. Figure 7 presents the GPS/INS integration system used in this study.

The EKF determines the general problem of trying to estimate the state of a discrete time controlled process that is clarified by the linear dynamic model:

$$
x_{k}=F_{k, k-1} x_{k-1}+G_{k-1} w_{k-1}
$$

with a measurement that is:

$$
z_{k}=H_{k} X_{k}+v_{k}
$$

where, $F_{k}, G_{k}$ and $H_{k}$ the state transition, the system noise coefficient and the design matrices, respectively. Also, $z_{k}$ and $x_{k}$ are the measurement and the error state vectors. The random variables $w_{k}$ and $v_{k}$ represent the system and measurement noises, respectively, with the uncorrelated and zero mean random processes:

$$
\begin{gathered}
E\left[w_{k} v_{j}^{\mathrm{T}}\right]=0 \\
w_{k} \sim N\left(0, Q_{k}\right) \\
v_{k} \sim N\left(0, R_{k}\right)
\end{gathered}
$$

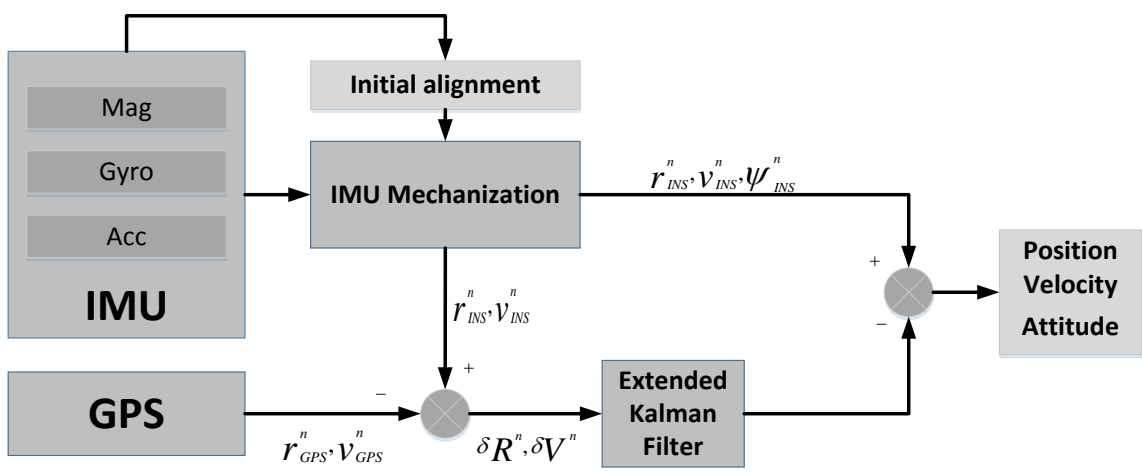

Figure 7. GPS/INS integration system used in this study. 
and the covariance matrices are:

$$
\begin{aligned}
& E\left[w_{k} w_{j}^{\mathrm{T}}\right]=\left\{\begin{array}{c}
Q_{k}, j=k \\
0, j \neq k
\end{array}\right. \\
& E\left[v_{k} v_{j}^{\mathrm{T}}\right]=\left\{\begin{array}{c}
R_{k}, j=k \\
0, j \neq k
\end{array}\right.
\end{aligned}
$$

In reality, the system noise covariance $Q_{k}$, and measurement noise covariance $R_{k}$ matrices might change with each time step. However, they are assumed to be constant. There are two steps in EKF process. The first step is the prediction of the system model:

$$
\begin{gathered}
\hat{x}_{\bar{k}}=F_{k, k-1} \hat{x}_{k-1} \\
P_{\bar{k}}=F_{k, k-1} P_{k-1} F_{k, k-1}^{\mathrm{T}}+G_{k-1} Q_{k-1} G_{k-1}^{\mathrm{T}}
\end{gathered}
$$

and the second step is the measurement update of the system model:

Kalman gain:

$$
\begin{gathered}
K_{k}=P_{\bar{k}} H_{k}^{\mathrm{T}}\left(H_{k} P_{\bar{k}} H_{k}^{\mathrm{T}}+R_{k}\right)^{-1} \\
P_{k}=\left(I-K_{k} H_{k}\right) P_{\bar{k}} \\
\hat{x}_{k}=\hat{x}_{\bar{k}}+K_{k}\left(z_{k}-H \hat{x}_{\bar{k}}\right)
\end{gathered}
$$

State update:

\subsection{Accident Detection}

Accident detection algorithm in this paper is an algorithm which can decide a low-speed car accident is happened or not. A low speed accident is an accident that the average speed is $3.5 \mathrm{~m} / \mathrm{s}$ and change in vehicle speed doesn't pass 6.5 $\mathrm{m} / \mathrm{s}$. A fatal accident that may terminate to serious injury and fatality, occur when a change in vehicle speed passes $7-12 \mathrm{~m} / \mathrm{s}$ in 1 second in any direction. In fact, the passenger airbags are triggered as soon as (45 $\mathrm{ms})$ the vehicle strongly collides an obstacle at $\delta \mathrm{V}=25-40 \mathrm{Km} / \mathrm{h}$. As this paper considers the lowspeed accident, the only acceleration is not sufficient parameter to detect the accident. So, another parameter which help us to detect the low-speed detection is curvature of the vehicle. Curvature of the vehicle is based on rotation speed of a vehicle which is shown with $\beta$ in Figure 8 . The parameter can be defined by:

$$
\beta=V / R
$$

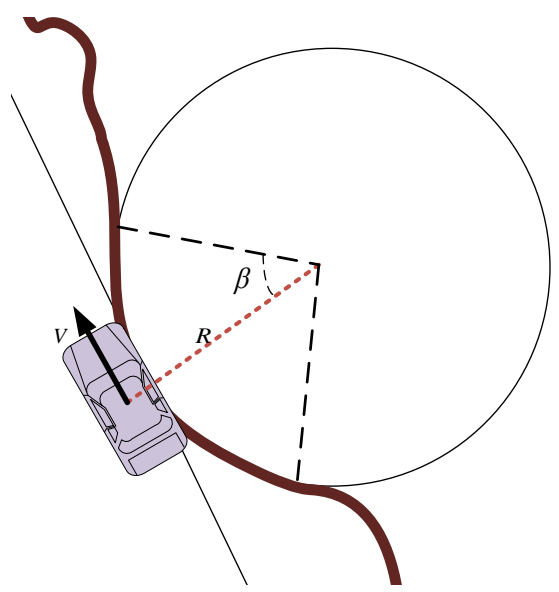

Figure 8. Concept of curvature of vehicle. 
where, $V$ is the angular velocity of the vehicle and $R$ is radius of the curve. So the curvature of the vehicle can be calculated by division of gyroscope value of $z$ axis to speed of the vehicle to obtain the rotational speed.

We defined two threshold-based filters for acceleration and curvature of vehicle to detect the low-speed accident. Figure 9 presents the flowchart of proposed low-speed accident detection. In first step attends the norm of acceleration in three axes is more than $3.7 \mathrm{~m} / \mathrm{s}^{2}$ and the second step considers the curvature of the vehicle is more than $15 \mathrm{1} / \mathrm{m}$. These threshold values were found based on different tests which are performed using Micro-IBB inside the vehicle in various maneuvers. After detection of collision, the position of the accident will be appeared based on proposed GPS/INS integration navigational system.

\section{Experimental Analysis, Results, and Discussion}

The data for analysis of the accident detection was obtained from the wireless Micro Intelligent Black Box (Micro-iBB), which was designed and assembled by the VTADS team of the LASSENA Lab in the ETS. Micro-iBB consists of a triad

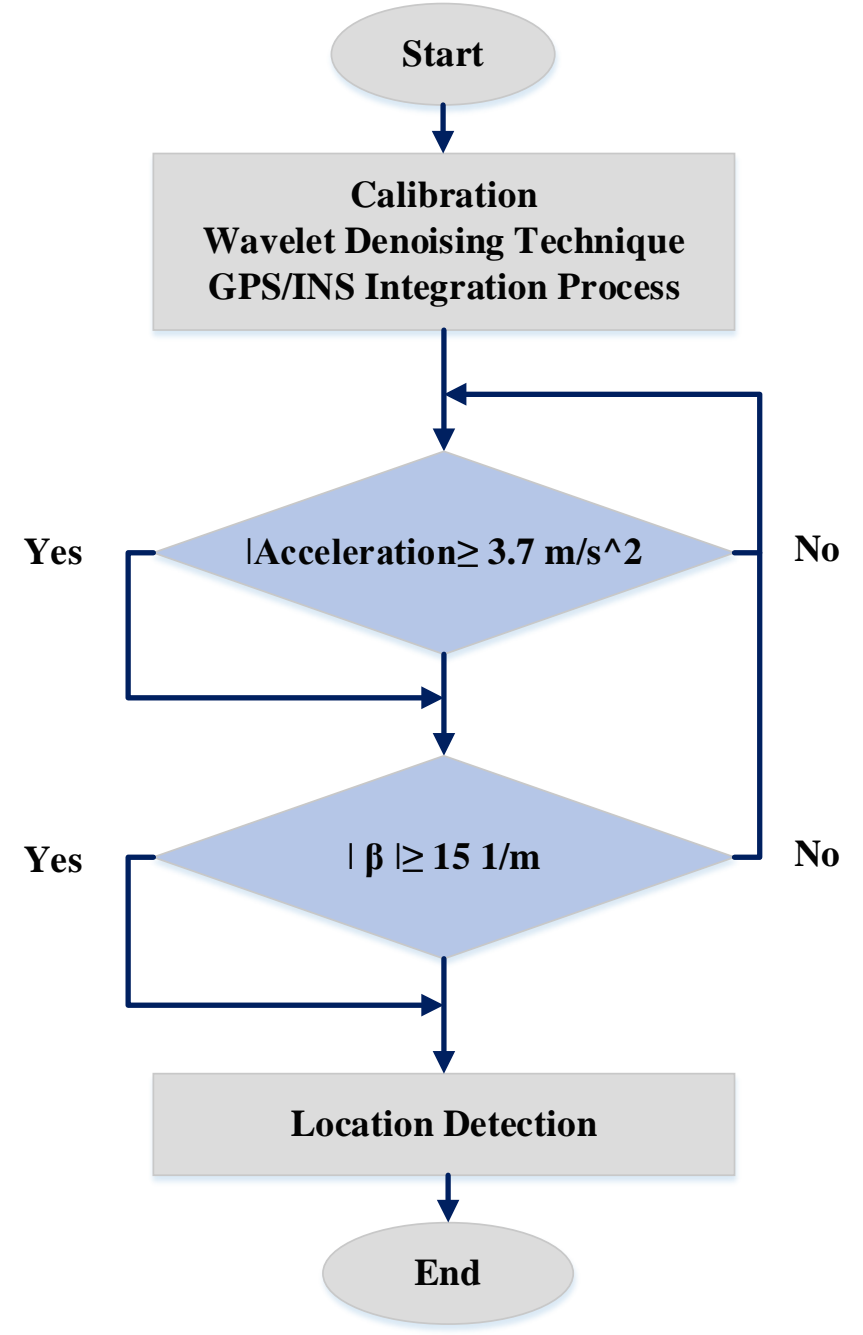

Figure 9. Flowchart of proposed low-speed accident detection. 
of accelerometers, gyroscopes, magnetometers and a temperature sensor. The serial number of the accelerometer, the gyroscope and the demonstration board are LSM303DLHC, L3GD20 and STEVAL-MKI119V1, respectively. The selected aiding devices in Micro-iBB consist of a GNSS receiver ( $u$-blox 7). Table 1 and Figure 10 present respectively the details of the Micro-iBB and its technical specification, which were used in this paper.

\subsection{Analysis of Wavelet Denoising Result}

When analyzing the signal of accelerometers or gyroscopes, it is important to isolate the stochastic process we want to observe. In fact, Gauss-Markov process occurs at low frequency on the inertial measurements. It is therefore important to filter the raw measurements to eliminate the high-frequency components contained on the signal. In order to do this, this paper proposes the use of a wavelet transform which eliminates the high-frequency noise components while retaining the relevant Gauss-Markov process information. Figure 11 shows the raw measurements from a low-cost accelerometer and gyroscopes before and after the use of a wavelet de-noising technique filter. This figure clearly illustrates the presence of high-frequency noise components on the initial raw signal, partially masking the Gauss-Markov process. Following the wavelet transform, the

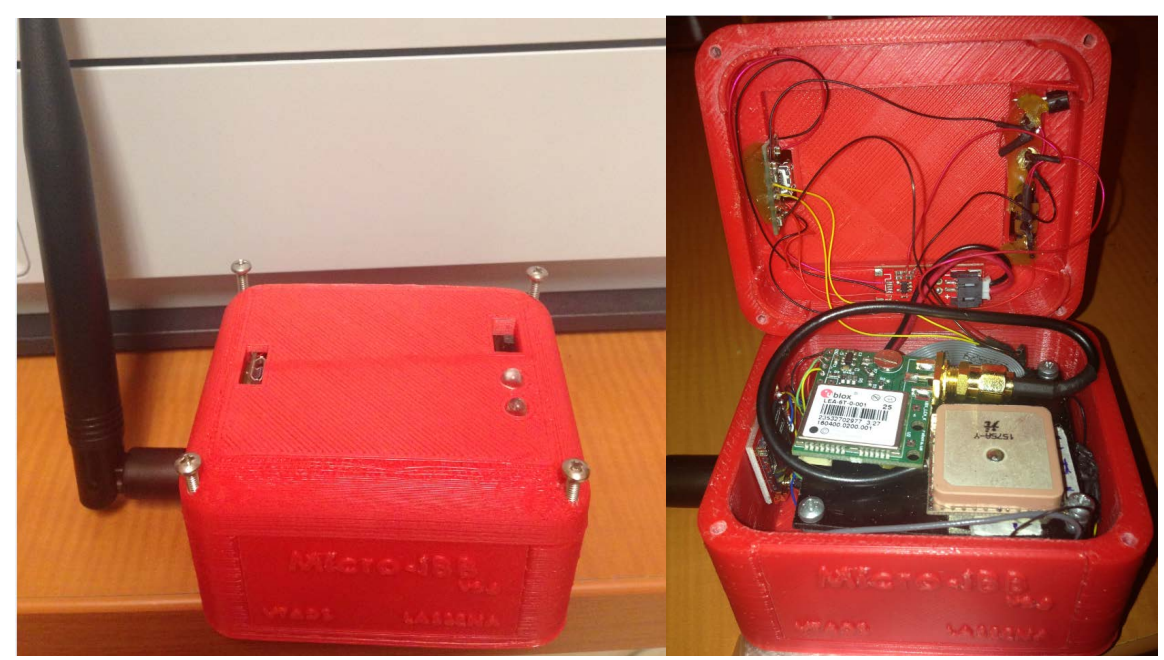

Figure 10. Wireless Micro intelligent Black Box (Micro-iBB).

Table 1. Technical specification of the angular rate and the acceleration sensors in MicroiBB.

\begin{tabular}{cccc}
\hline \multicolumn{2}{c}{ Accelerometer } & \multicolumn{2}{c}{ Gyroscope } \\
\hline Data Rate & $200 \mathrm{~Hz}$ & Data Rate & $200 \mathrm{~Hz}$ \\
Temperature Range & $-40 \sim+85^{\circ} \mathrm{C}$ & Temperature Range & $-40 \sim+85^{\circ} \mathrm{C}$ \\
Input range & $\pm 2 \mathrm{~g}$ & Input range & $\pm 250^{\circ} / \mathrm{sec}$ \\
Linear sensitivity & $2 \mathrm{mg} / \mathrm{LSB}$ & Sensitivity & $\mathrm{m} \% \mathrm{sec} / \mathrm{digit}$ \\
& & Rate noise density & $0.03 \% / \mathrm{sec} / \sqrt{\mathrm{Hz}}$ \\
Acceleration noise density & $220 \mu \mathrm{g} / \sqrt{\mathrm{Hz}}$ & Nonlinearity & $0.2 \% \% \mathrm{sec}$ \\
\hline
\end{tabular}



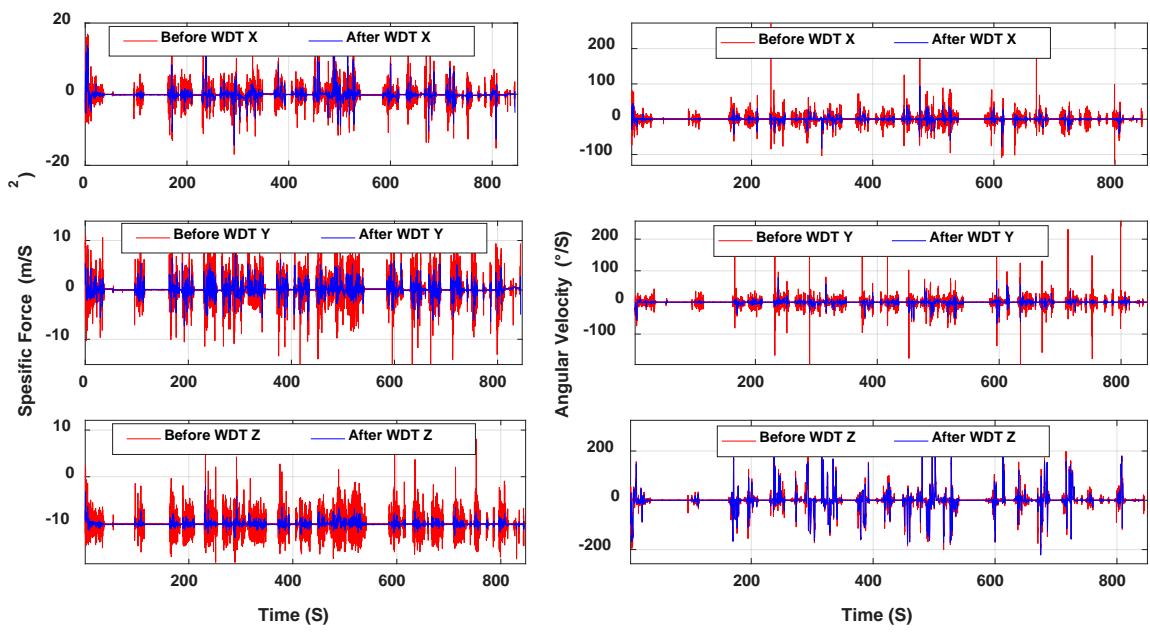

Figure 11. Raw measurements accelerometer and gyroscope before and after applied WDT.

high-frequency components are greatly attenuated and the Gauss-Markov process becomes more evident. It is on this filtered signal that the autocorrelation function must be applied so that the characteristic curve obtained is sufficiently defined to be able to extract the different parameters.

\subsection{Results of Proposed Calibration Method}

The calibration algorithm developed in this paper were used to determine the initial calibration parameters of the units shown in Figure 10. The calibration algorithm presented in section 3.1 is based on the multi-position method, so that the measurements from each of the inertial units are recorded according to different orientations. When recording the measurements, the inertial units are arranged inside its supporter in the form of a square prism. This makes it possible to eliminate the irregular surfaces of these sensors and facilitate the taking of flat measurements. For the tests presented in this section, five different tests staggered over a duration of 5 seconds; were realized and processed by the proposed calibration algorithm.

The calibration of the accelerometers is based on the observation of the gravity vector which is used as a reference within the algorithm. The theoretical components of this vector are calculated using the gravity model; and are considered to be constant throughout the calibration procedure. In stationary conditions and in the absence of error, the absolute value from the accelerometers should be equal to that of the theoretical gravity vector. However, when the measurements are contaminated, this assumption is no longer valid and the measured standard can be quite different from the reference value. The calibration of the gyroscopes is the same as accelerometers.

The raw measurements from the accelerometers/gyroscopes before and after calibration are shown in Figure 12. By analyzing these figures, it can be seen that for the low-cost inertial units the absolute value varies significantly according to the orientation of the inertial unit. This behavior results directly from the pres- 
ence of large scale factor errors and misalignment on the measurements of these IMUs. Also, this figure conform that calibration process can definitely reduce the systematic errors of the accelerometers and gyroscopes measurements.

Calculated parameters of calibration in 5 different test case as long as mean and variance of the estimated parameters of calibration are presented in Table 2 and Table 3. Table 2 shows that the accelerometer errors are around 7.2 - 32.4 $\mathrm{mg}$ for bias parameters, around $0.13 \%-3.9 \%$ for scale-factor and misalignment parameters. Table 3 presents the existence of a neglect able bias error around 3.4 $\times 10^{-3}-10.9 \times 10^{-3} \mathrm{rad} / \mathrm{s}$. These results confirm that the calibration process is inevitable for low-cost sensors.

\subsection{Results of GPS/INS Integration and Accident Detection}

To evaluate the performance of the algorithms carried out in this paper, two different scenarios were developed. For demonstration, the loosely coupled GPS/

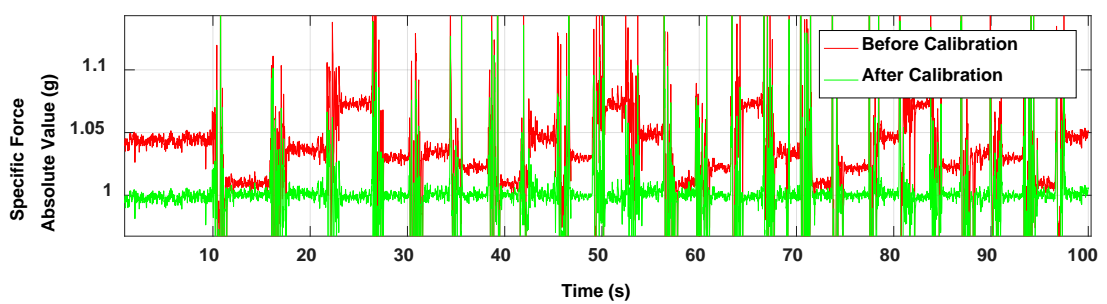

(a)

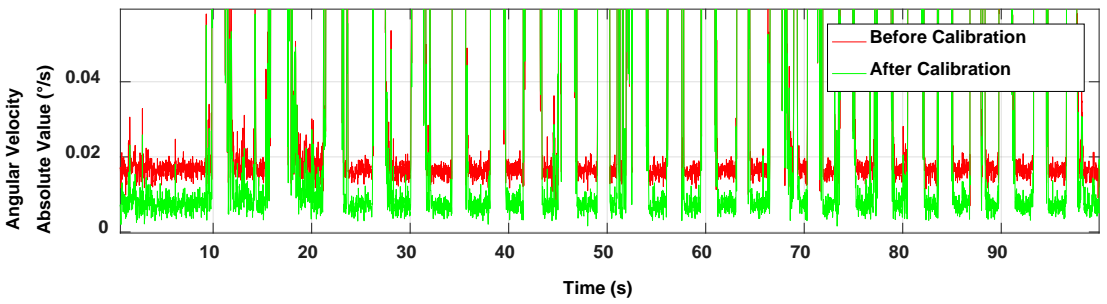

(b)

Figure 12. Absolute values before and after calibration for (a) accelerometers and (b) gyroscopes.

Table 2. Results of accelerometer calibration in 5 test cases.

\begin{tabular}{cccccccc}
\hline Parameters & $\# 1$ & $\# 2$ & $\# 3$ & $\# 4$ & $\# 5$ & $\mu$ & $\sigma^{2}$ \\
\hline$S F_{x}^{i}$ & 0.9616 & 0.9602 & 0.9602 & 0.9605 & 0.9610 & 0.9607 & $3.54 \mathrm{e}-07$ \\
$-S F_{y}^{i} M_{z}^{i}$ & -0.0274 & 0.0130 & 0.0019 & 0.0013 & 0.0373 & 0.0052 & $5.45 \mathrm{e}-04$ \\
$+S F_{z}^{i} M_{y}^{i}$ & -0.036 & -0.0284 & -0.0011 & -0.0098 & -0.0257 & -0.0203 & $2.06 \mathrm{e}-04$ \\
$S F_{y}^{i}$ & 0.9632 & 0.9502 & 0.9751 & 0.9751 & 0.9738 & 0.9675 & $1.18 \mathrm{e}-04$ \\
$-S F_{y}^{i} M_{x}^{i}$ & 0.0058 & -0.0399 & -0.0044 & -0.0039 & 0.0116 & -0.0061 & $4.02 \mathrm{e}-04$ \\
$S F_{z}^{i}$ & 0.9641 & 0.9586 & 0.9628 & 0.9623 & 0.9629 & 0.9622 & $4.50 \mathrm{e}-06$ \\
$B_{x}^{i}$ & -0.0322 & -0.0325 & -0.0316 & -0.0317 & -0.0338 & -0.0324 & $7.80 \mathrm{e}-07$ \\
$B_{y}^{i}$ & 0.0084 & -0.0316 & -0.0046 & -0.0042 & -0.0043 & -0.0072 & $2.17 \mathrm{e}-04$ \\
$B_{z}^{i}$ & 0.0177 & 0.001 & 0.0005 & 0.0055 & 0.0293 & 0.0109 & $1.52 \mathrm{e}-04$ \\
\hline
\end{tabular}


Table 3. results of gyroscopes calibration in 5 test cases.

\begin{tabular}{cccccccc}
\hline Parameters & $\# 1$ & $\# 2$ & $\# 3$ & $\# 4$ & $\# 5$ & $\mu$ & $\sigma^{2}$ \\
\hline$S F_{x}^{i}$ & 0.9995 & 0.9999 & 0.9998 & 0.9995 & 0.99995 & 0.9997 & $3.28 \mathrm{e}-08$ \\
$-S F_{y}^{i} M_{z}^{i}$ & -0.0000 & 0.0000 & 0.0000 & -0.0000 & 0.0000 & $-9.28 \mathrm{e}-06$ & $2.28 \mathrm{e}-10$ \\
$+S F_{z}^{i} M_{y}^{i}$ & 0.0000 & -0.0000 & 0.0000 & 0.0000 & -0.0000 & $3.36 \mathrm{e}-06$ & $2.75 \mathrm{e}-11$ \\
$S F_{y}^{i}$ & 0.9984 & 0.9999 & 0.9998 & 0.9993 & 0.9999 & 0.9995 & $3.76 \mathrm{e}-07$ \\
$-S F_{y}^{i} M_{x}^{i}$ & -0.0000 & 0.0000 & 0.0000 & 0.0000 & 0.0000 & $2.11 \mathrm{e}-06$ & $1.28 \mathrm{e}-11$ \\
$S F_{z}^{i}$ & 0.9995 & 0.9999 & 0.9998 & 0.9999 & 0.9999 & 0.9998 & $2.27 \mathrm{e}-08$ \\
$B_{x}^{i}$ & 0.0077 & 0.0045 & 0.0034 & 0.0036 & 0.0045 & 0.0048 & $3.01 \mathrm{e}-06$ \\
$B_{y}^{i}$ & -0.0131 & -0.0114 & -0.0083 & -0.0089 & -0.0127 & -0.0109 & $4.85 \mathrm{e}-06$ \\
$B_{z}^{i}$ & -0.0035 & -0.0039 & -0.0029 & -0.0060 & -0.0008 & -0.0034 & $3.48 \mathrm{e}-06$ \\
\hline
\end{tabular}

INS integration without augmentation was utilized in a three-dimensional navigation system. In the loosely coupled GPS/INS integration, position and velocity observed by GPS are the auxiliary measurements. So, the measurement model can be as follows

$$
\delta \mathrm{z}=H \delta \bar{x}+\eta
$$

where, $H$ is $\left[\begin{array}{ccc}H_{R} & 0_{3 \times 3} & 0_{3 \times 2} \\ 0_{3 \times 3} & H_{R} & 0_{3 \times 2}\end{array}\right]$, and $H_{R}=H_{R}=I_{3 \times 3}$. Measurement noise vector $\eta$ is $\left[\begin{array}{ll}\eta_{R} & \eta_{V}\end{array}\right]^{\mathrm{T}}$, denoting the position and velocity noise. Vector $\eta$ is defined by measurement noise model $R$, where

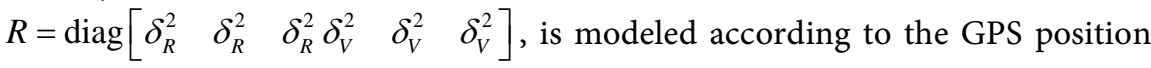
and velocity uncertainty with zero mean and covariance matrix. The error states vector, relating to the navigation system of this study, are as follows:

$$
\delta \bar{x}=\left[\begin{array}{lll}
\delta \bar{R} & \delta \bar{V} & \delta \bar{\psi}
\end{array}\right]^{\mathrm{T}}
$$

where, $\delta \bar{R}$ and $\delta \bar{V}$ are respectively $\left[\begin{array}{lll}\delta R_{N} & \delta R_{E} & \delta R_{U}\end{array}\right]$ and $\left[\begin{array}{lll}\delta V_{N} & \delta V_{E} & \delta V_{U}\end{array}\right]$, denoting position and velocity residual errors in East North $U_{p}(\mathrm{ENU})$ frame; $\delta \bar{\psi}$ is $\left[\begin{array}{llll}\delta \psi_{N} & \delta \psi_{E} & \delta \psi_{U}\end{array}\right]$ is the attitude error. The proposed land-navigation solution was implemented with an experimental setup for a road-test. The tests were performed by Micro-IBB, whose specifications are detailed under Section 4.1. The results were evaluated against a reference solution provided by Novatel SPAN technology. It is consisting of Novatel receiver and CPT tactical-grad IMU to validate the proposed method and to assess the overall performance during the GPS outages. Figure 13 and Figure14 present the hardware of the reference and the vehicle used in test cases.

The raw data of the route-test was gathered from different sensors of MicroiBB. The first trajectory was figured out in ideal environment to evaluate the accident detection algorithm. The second trajectory was performed in different environmental situations to show how it can satisfy the overall quality and reliability requirements of navigational systems. The hardware architecture used for each of the two test scenarios is the same. 


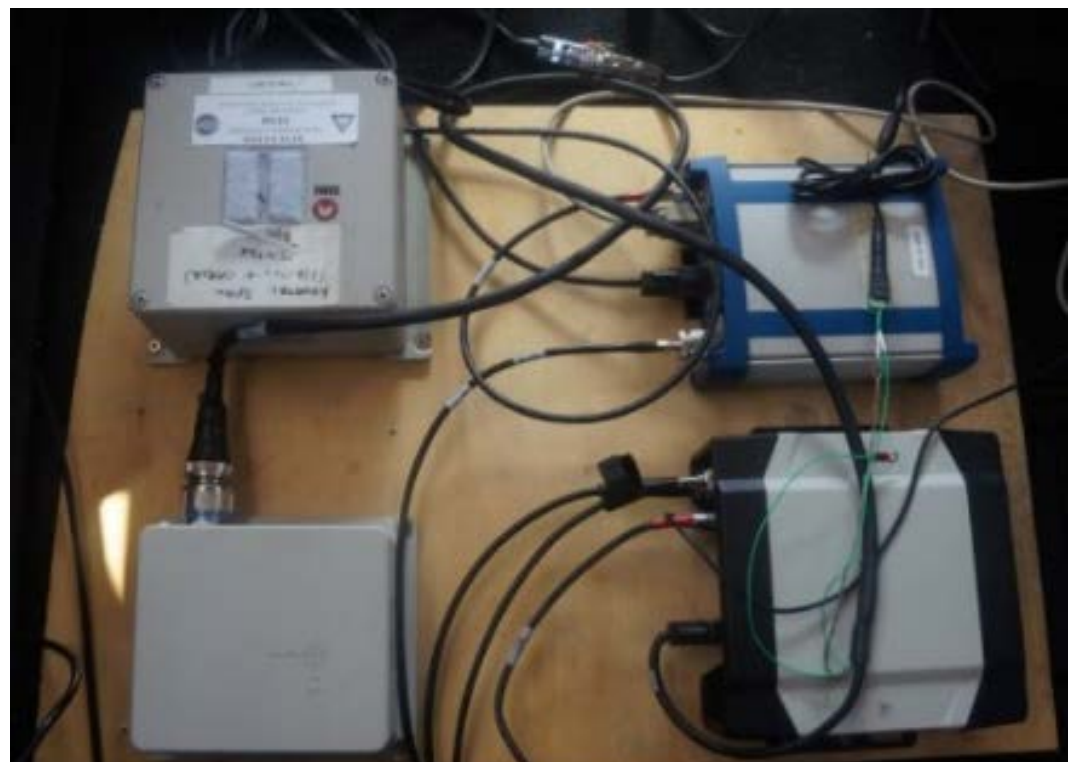

Figure 13. Real hardware architecture used for dynamic testing as the reference.

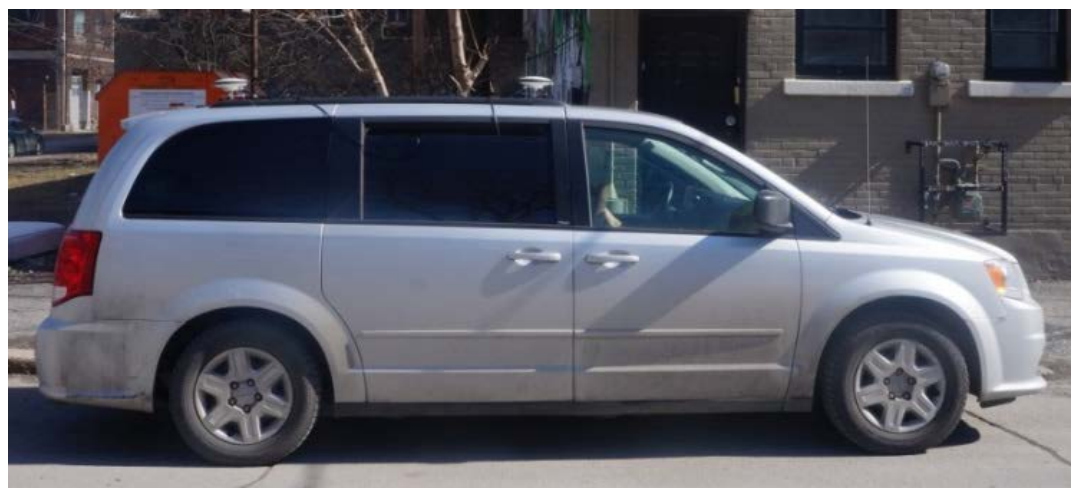

Figure 14. Vehicle of the test.

\subsubsection{First Scenario: Ideal Environment}

The scenario in ideal environment was chosen in order to allow the GPS receiver to obtain optimum visibility throughout the trajectory or to have at least 6 satellites in direct line of sight at all times. This scenario thus provides a control navigation solution for the different algorithms allowing to quantify their performances in an ideal environment. The ideal environment is chosen to show the behaviour of accident detection algorithm regardless of GPS blockage areas. The trajectory was carried out on the Griffintown of Montréal, specifically in the municipalities of Chalet de Mont-Royal. This region is mainly composed of residential districts and is remote from skyscrapers, dense forest areas and any other difficult environment that may disrupt the navigation solution. This trajectory takes around 3 hours and half of driving considering more than 20 times simulated accident during the trajectory.

Figure 15 shows the velocity and differential velocity of the car. As you can see, the speed of the car is less than $20 \mathrm{~m} / \mathrm{s}$ during the trajectory. This speed was considered in this study, as we need to detect the low-speed accident. Figure 16 shows the acceleration in each axes as long as nom of acceleration during the 
trajectory. Figure 16 presents that the acceleration in $y$-axis has the maximum impact to detect the acceleration as it has the maximum value compared to the two other axes. It should be mentioned that the acceleration in $z$ axis is presented regardless of gravity force of Earth.

Figure 17 depicts the curvature and differential curvature of the vehicle. As it can show the movement of vehicle after big acceleration detection, it plays critical role in low-speed accident detection. Figure 18 presents the accident detection algorithms based on both acceleration and curvature of the moving vehicle. According to proposed model in accident detection which is explained in section 3.3 if the absolute value of acceleration is more than $3.7 \mathrm{~m} / \mathrm{s}^{-2}$ and curvature of the vehicle is more than $15^{\circ}$ an accident is detected. After the accident is detected, the position of the accident reveals on the map. Figure 19 presents the trajectory in the first scenario. The red spot in the map shows the position of the first accident detected during this trajectory.
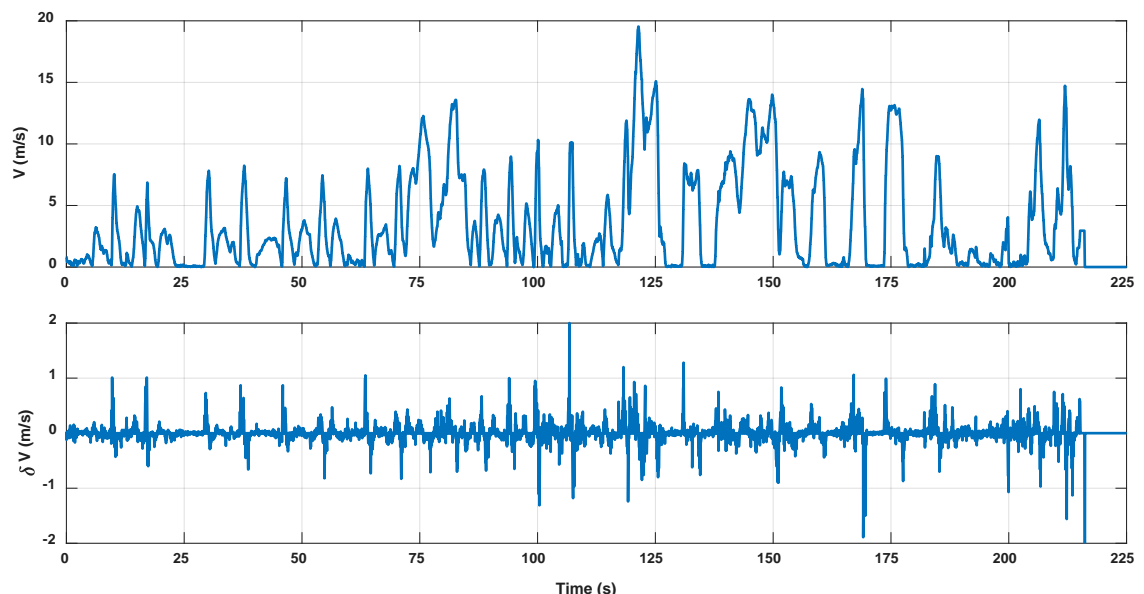

Figure 15. Vehicle speed in the first scenario.
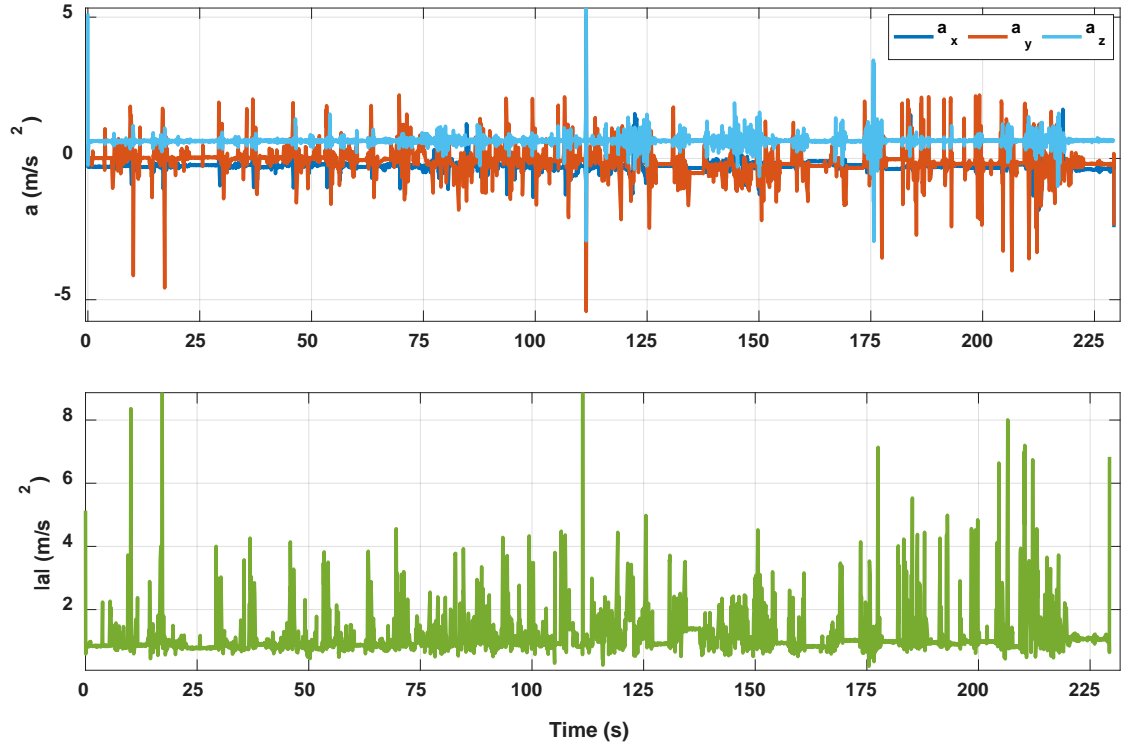

Figure 16. Acceleration of the vehicle in the first scenario. 

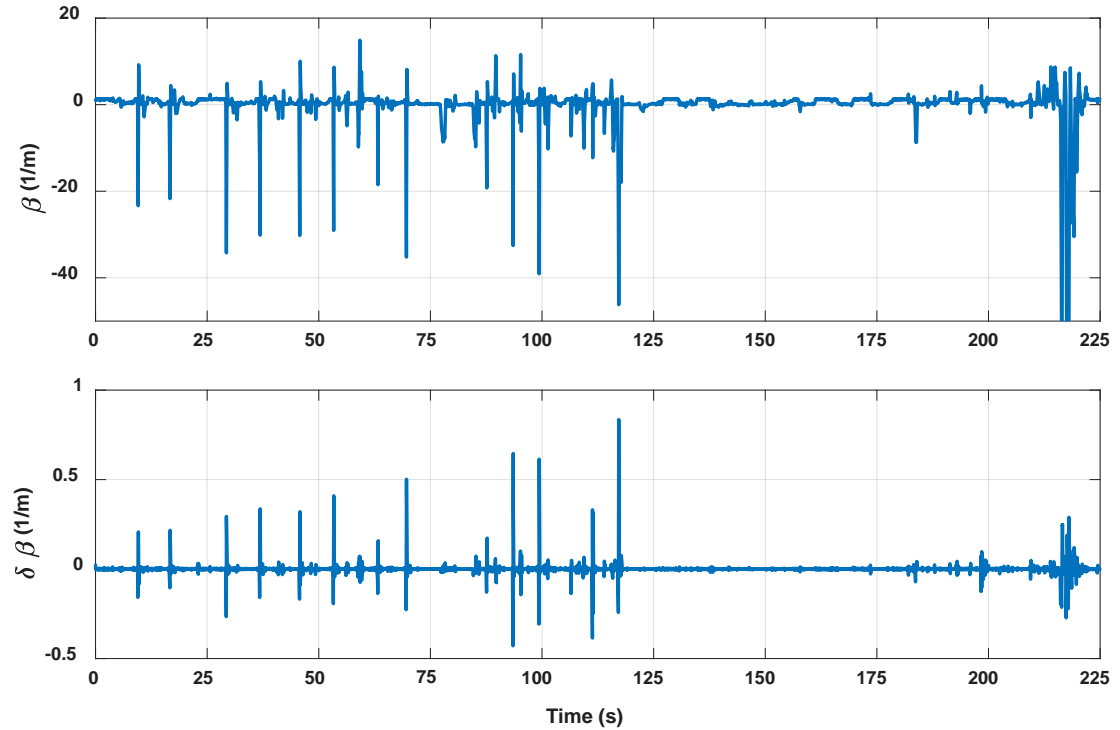

Figure 17. Curvature of the vehicle in the first scenario.
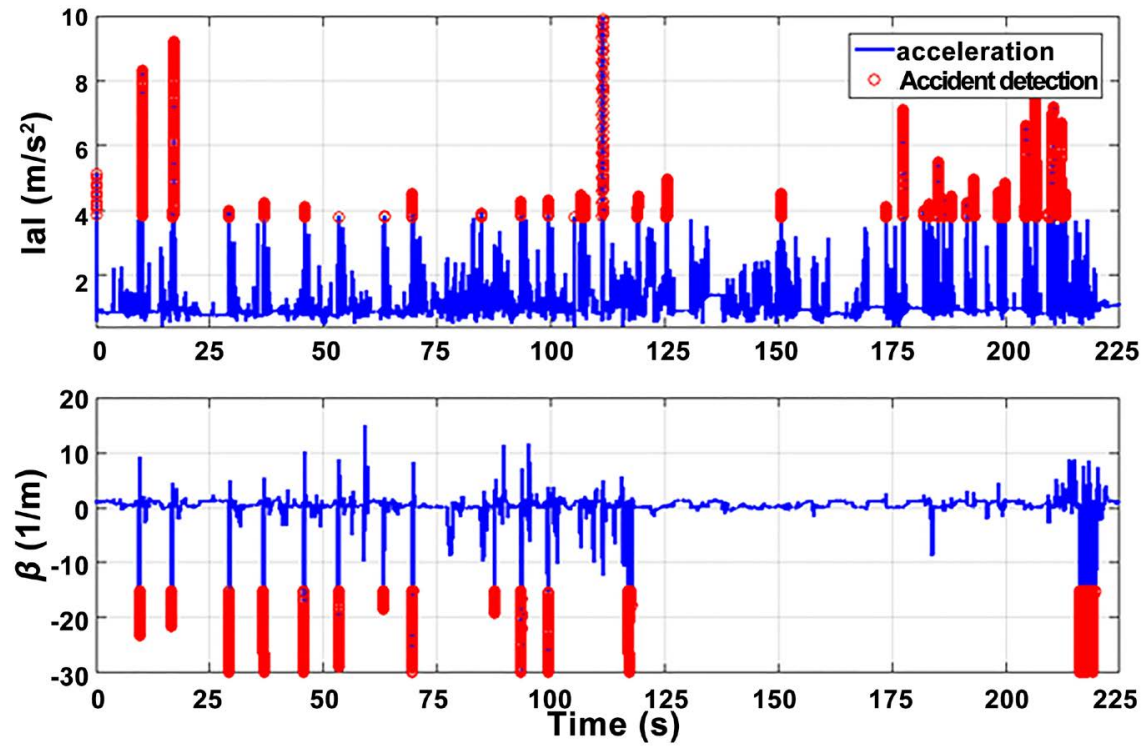

Figure 18. Accident detection based on acceleration and curvature of the vehicle.

\subsubsection{Second Scenario: Harsh Environment}

The second trajectory was figured out in an urban freeway, which allowed the authors to drive at high speed. Also, it was continued in downtown area that contains numerous prominent skyscrapers. The scenario in difficult environment was carried out in order to evaluate the impact of such an environment on the autonomous GPS navigation solution as well as the real contributions of the various algorithms developed. Before each test, a rigorous initial calibration and alignment procedure was performed for each of the inertial units used. The SPAN system is also used as the reference.

This scenario can be characterized by four distinct portions, namely a portion made of a severe urban canyon in the Old Port of Montreal, a portion of Highway 134 and Highway 15 comprising several tunnels and viaducts, and an open 
section on Jean-Talon Street and on Côte-des-Neiges route as well as a second section of a severe urban canyon in downtown Montreal (see Figure 20). The trajectory was carried out in downtown Montreal and consists of narrow urban canyons, tunnels, and viaducts. The total distance is $27.4 \mathrm{~km}$, and it takes $1 \mathrm{~h} 12$ $\min (55 \mathrm{~min}$ without traffic) to finish the trajectory.

Figure 21 illustrates the horizontal positioning results for whole the trajectory of both algorithm configurations of GPS/INS integration alone and GPS/INS integration with applying the proposed Powel Dog-leg's method calibration. In this figure green color presents the solution od Novatel SPAN as the reference, light blue color dots present the GPS alone solution; red and blue colors show

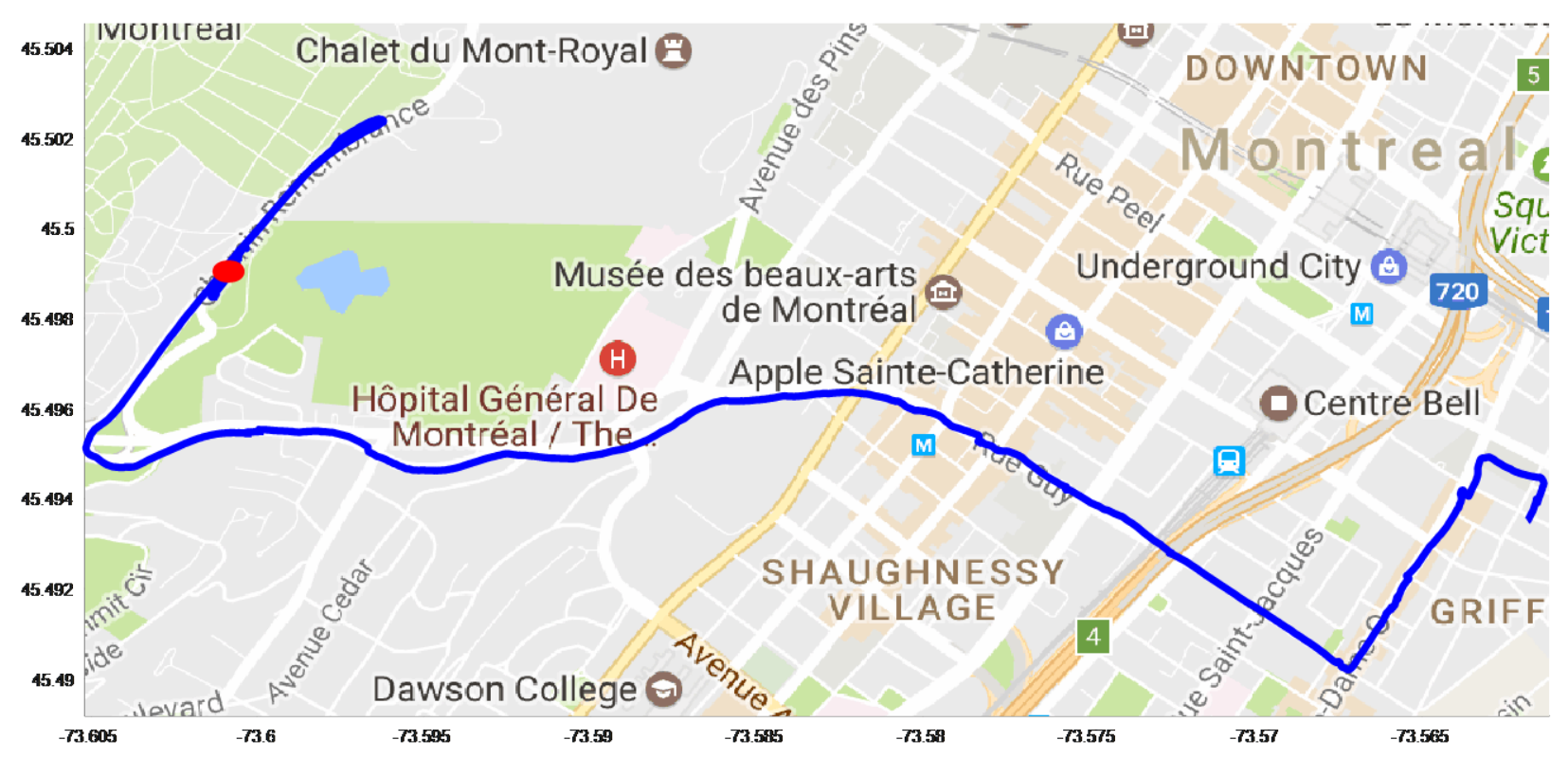

Figure 19. Position detection of the first accident in the first scenario.

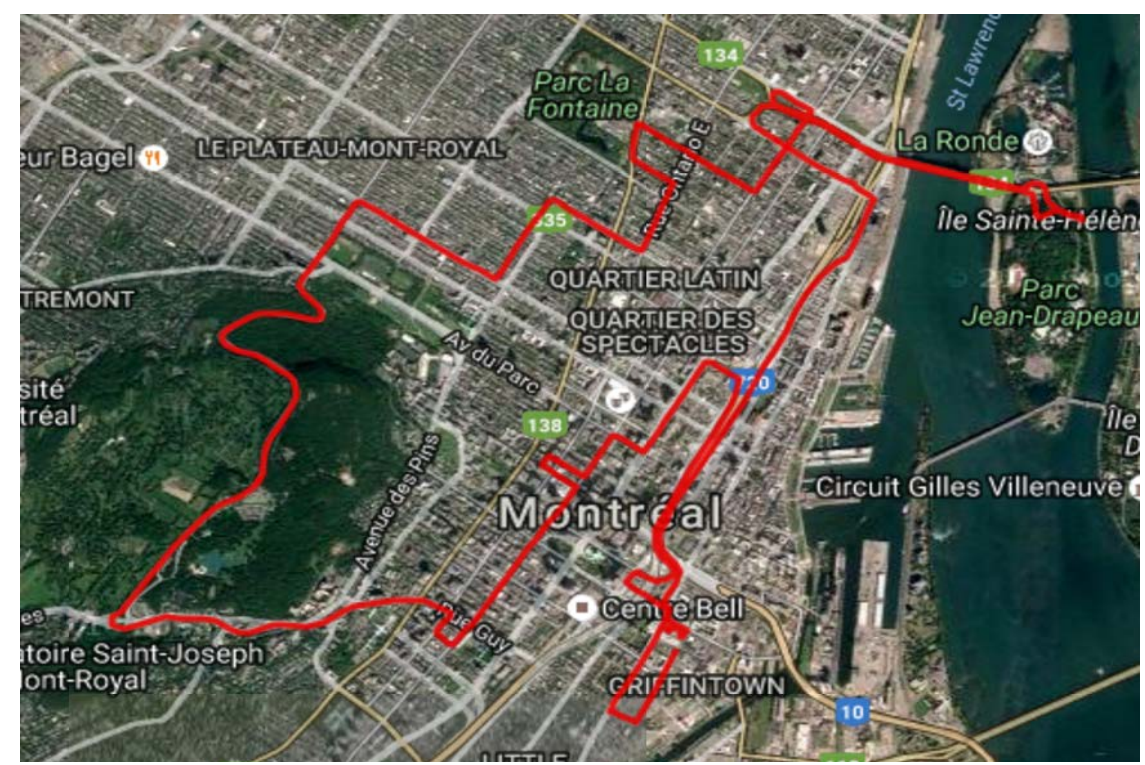

Figure 20. Overview of the scenario in harsh environment. 
the GPS/INS integration solution alone and GPS/INS integration solution after applying the calibration procedure.

Figure 22 shows the horizontal positioning results of both solutions for the four critical selected areas compared to the Novatel SPAN reference solution. Areas \#2 and 3 have interrupted GPS coverage however areas \#1 and 4 consist of fully GPS outages. These figures obviously illustrate the significant improvement of the navigational solution with using calibrated sensors' measurements in harsh urban canyons.

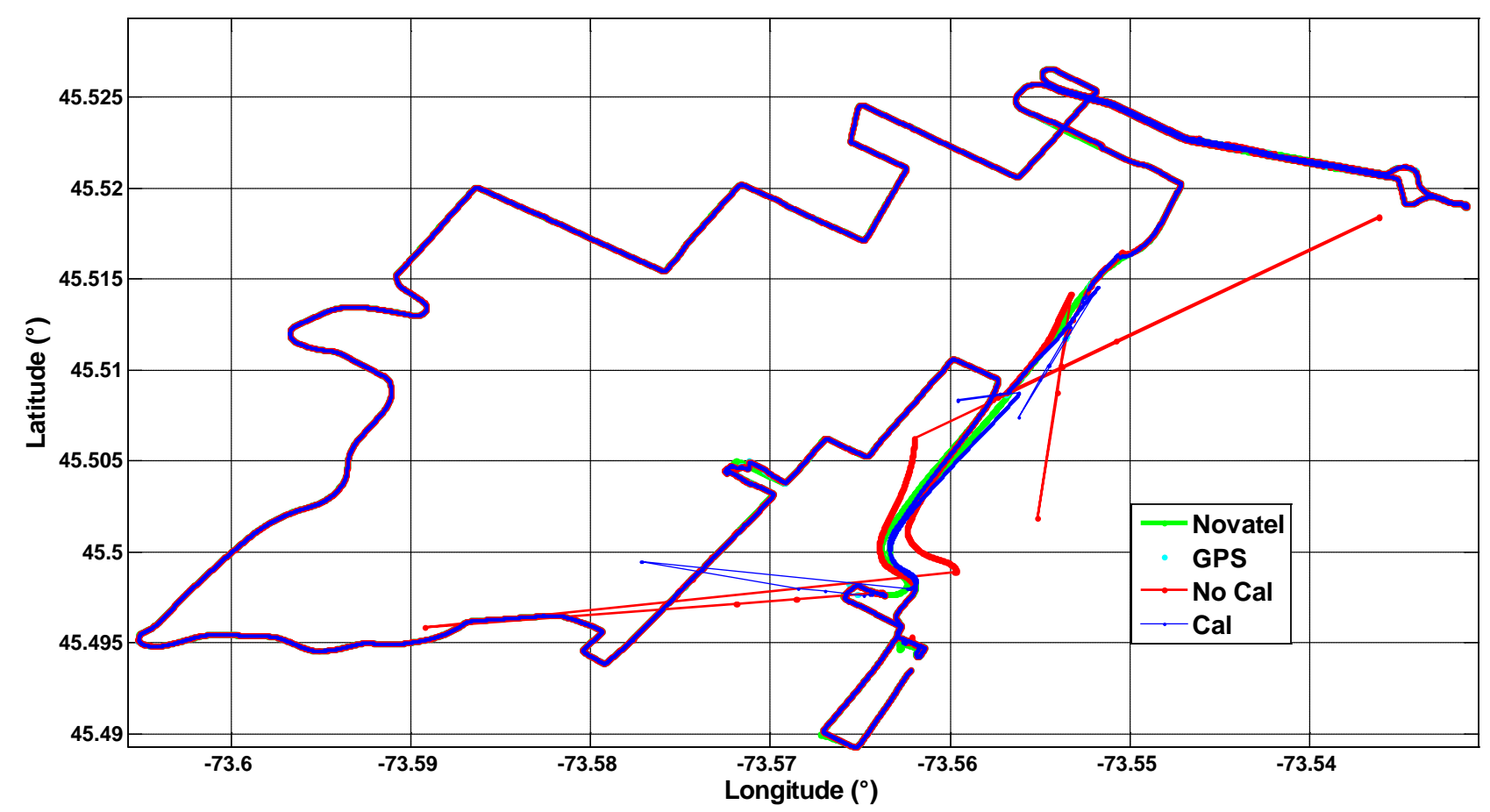

Figure 21. Comparison of GPS/INS integration with/without calibration.
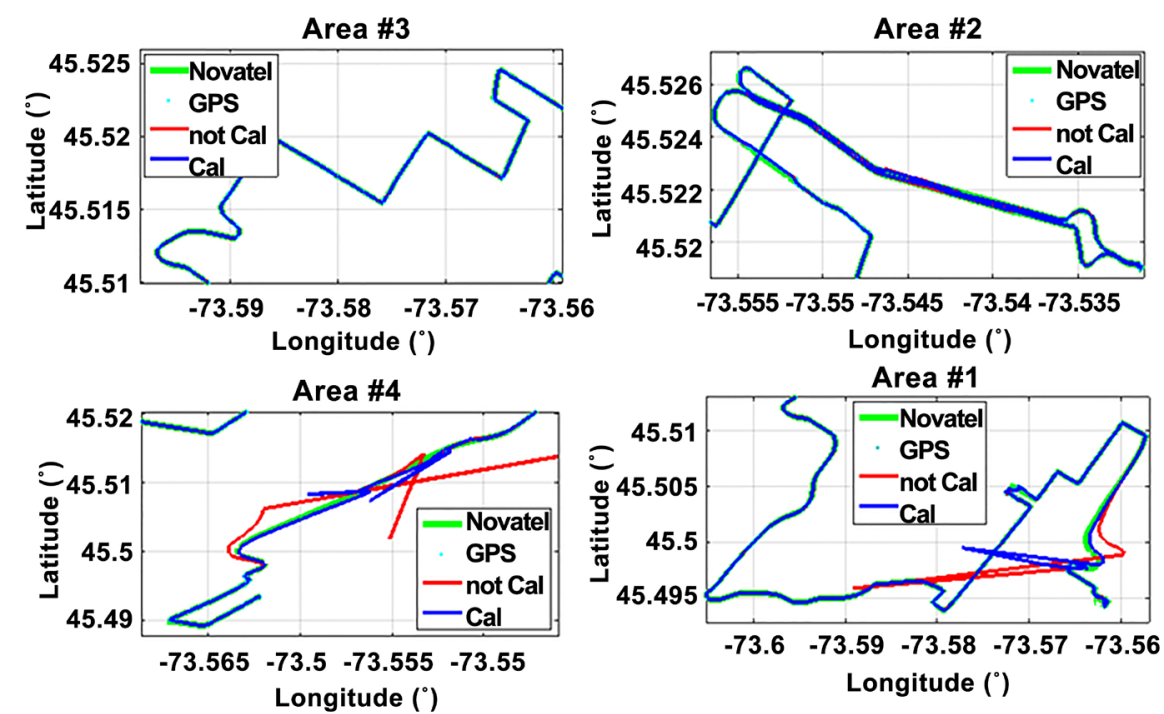

Figure 22. Comparison of GPS/INS integration with/without calibration for the four selected critical areas. 


\section{Conclusion}

This paper provided a solution for the analysis and the diagnosis of the car accident and Location-of-collision detection system based on GPS/INS integration. The solution uses the functions of vehicle dynamics, expressed by a set of raw measurements. These raw measurements are obtained from various sensors. This solution can characterize the car accident based on the capabilities of intelligent systems. The models consider GPS/INS-based navigation algorithm, calibration of navigational sensors, a de-nosing method as long as vehicle accident. In addition, the location-based accident detection model is tested in different scenarios. The results illustrate that under harsh environments with no GPS signal, location of accident can be detected. Also results confirm that calibration of sensors has an important role in position correction algorithm. Finally, the results presents that the proposed accident detection algorithm can recognize accidents and related its positions.

\section{Acknowledgements}

This research is part of the project entitled "VTADS: Vehicle Tracking and Accident Diagnostic System". It is supported by the Natural Sciences and Engineering Research Council of Canada (NSERC) and École de Technology Supérieure (LASSENA Lab), in collaboration with two industrial partners, namely iMetrik Global Inc. and Future Electronics.

\section{References}

[1] Consulting, R.S.C. (2011) Road Safety in Canada. Public Health Agency of Canada.

[2] Landry, R.J. (2012) Vehicle Tracking and Accident Diagnostic System (VTADS).

[3] Li, N. and Busso, C. (2013) Analysis of Facial Features of Drivers under Cognitive and Visual Distractions. 2013 IEEE International Conference on Multimedia and Expo (ICME), San Jose, 15-19 July 2013, 1-6. https://doi.org/10.1109/icme.2013.6607575

[4] Zongyuan, W., Huanzhang, L., Shuanghong, Z. and Feng, S. (2014) The Study of Square Root Cubature Kalman Smoother and Its Application on INS/GPS Integrated Navigation. 2014 IEEE International Conference on Mechatronics and $\mathrm{Au}$ tomation (ICMA), Tianjin, 3-6 August 2014, 1827-1832. https://doi.org/10.1109/ICMA.2014.6885979

[5] Zhao, L., Qiu, H. and Feng, Y. (2014) Study of Robust Filtering Application in Loosely Coupled INS/GPS System. Mathematical Problems in Engineering, 2014, Article ID: 904062

[6] Jorgensen, M.J., Paccagnan, D., Poulsen, N.K. and Larsen, M.B. (2014) IMU Calibration and Validation in a Factory, Remote on Land and at Sea. Position, Location and Navigation Symposium-PLANS, Monterey, 5-8 May 2014, 1384-1391.

[7] Zhang, R., Hoflinger, F. and Reind, L.M. (2014) Calibration of an IMU Using 3-D Rotation Platform. Sensors Journal, 14, 1778-1787. https://doi.org/10.1109/JSEN.2014.2303642

[8] Bertsekas, D.P. (1996) Incremental Least Squares Methods and the Extended Kalman Filter. SIAM Journal on Optimization, 6, 807-822.

https://doi.org/10.1137/S1052623494268522 
[9] Dorveaux, E., Vissiere, D., Martin, A.-P. and Petit, N. (2009) Iterative Calibration Method for Inertial and Magnetic Sensors. Proceedings of the 48 th IEEE Conference on Decision and Control, Shanghai, 15-18 December 2009, 8296-8303. https://doi.org/10.1109/cdc.2009.5399503

[10] Noureldin, A., Karamat, T.B., Eberts, M.D. and El-Shafie, A. (2009) Performance Enhancement of MEMS-Based INS/GPS Integration for Low-Cost Navigation Applications. IEEE Transactions on Vehicular Technology, 58, 1077-1096. https://doi.org/10.1109/TVT.2008.926076

[11] Kamiński, T., Niezgoda, M., Kruszewski, M., Grzeszczyk, R., Drop, T. and Filipek, P. (2012) Collision Detection Algorithms in the eCall System. Journal of KONES, 19, 267-274.

[12] Nassar, S. (2003) Improving the Inertial Navigation System (INS) Error Model for INS and INS/DGPS Applications. Department of Geomatics Engineering, University of Calgary, Calgary.

[13] Ban, Y., Niu, X., Zhang, T., Zhang, Q., Guo, W. and Zhang, H. (2014) Low-End MEMS IMU Can Contribute in GPS/INS Deep Integration. Position, Location and Navigation Symposium-PLANS, Monterey, 5-8 May 2014, 746-752. https://doi.org/10.1109/plans.2014.6851440

[14] Quinchia, A.G., Falco, G., Falletti, E., Dovis, F. and Ferrer, C. (2013) A Comparison between Different Error Modeling of MEMS Applied to GPS/INS Integrated Systems. Sensors, 13, 9549-9588. https://doi.org/10.3390/s130809549

[15] Kang, C.H., Kim, S.Y. and Park, C.G. (2011) Improvement of a Low Cost MEMS Inertial-GPS Integrated System Using Wavelet Denoising Techniques. International Journal of Aeronautical and Space Sciences, 12, 371-378. https://doi.org/10.5139/IJASS.2011.12.4.371

[16] El-Sheimy, N., Nassar, S. and Noureldin, A. (2004) Wavelet De-Noising for IMU Alignment. Aerospace and Electronic Systems Magazine, 19, 32-39. https://doi.org/10.1109/MAES.2004.1365016

[17] Schmidt, G.T. and Phillips, R.E. (2010) INS/GPS Integration Architectures. DTIC Document.

[18] Falco, G., Einicke, G.A., Malos, J.T. and Dovis, F. (2012) Performance Analysis of Constrained Loosely Coupled GPS/INS Integration Solutions. Sensors, 12, 1598316007. https://doi.org/10.3390/s121115983

[19] Ryan, J.G. and Bevly, D.M. (2014) On the Observability of Loosely Coupled Global Positioning System/Inertial Navigation System Integrations with Five Degree of Freedom and Four Degree of Freedom Inertial Measurement Units. Journal of Dynamic Systems, Measurement, and Control, 136, Article ID: 021023. https://doi.org/10.1115/1.4025985

[20] Skog, I. and Handel, P. (2011) Time Synchronization Errors in Loosely Coupled Gps-Aided Inertial Navigation Systems. IEEE Transactions on Intelligent Transportation Systems, 12, 1014-1023. https://doi.org/10.1109/TITS.2011.2126569

[21] Bar-Shalom, Y., Li, X.R. and Kirubarajan T. (2004) Estimation with Applications to Tracking and Navigation: Theory Algorithms and Software. John Wiley \& Sons, Hoboken.

[22] Škaloud, J. (1999) Optimizing Georeferencing of Airborne Survey Systems by INS/ DGPS. University of Calgary, Calgary.

[23] Foxlin, E. and Naimark, L. (2003) Miniaturization, Calibration \& Accuracy Evaluation of a Hybrid Self-Tracker. The 2 nd IEEE and ACM International Symposium on Mixed and Augmented Reality, Tokyo, 7-10 October 2003, 151-160. 
https://doi.org/10.1109/ISMAR.2003.1240698

[24] Fang, B., Chou, W. and Ding, L. (2014) An Optimal Calibration Method for a MEMS Inertial Measurement Unit. International Journal of Advanced Robotic Systems, 11, 1-14. https://doi.org/10.5772/57516

[25] Lavoie, P. and Landry, R.J. (2013) Sensor Error Compensation Methods for Performance Enhancement of a Low-Cost INS/GPS Navigation Algorithm Used in Severe Urban Environments. Proceedings of the ION 2013 Pacific PNT Meeting, Honolulu, 23-25 April 2013, 207-220.

[26] Skog, I. and Händel, P. (2006) Calibration of a MEMS Inertial Measurement Unit. The 17 th IMEKO WORLD CONGRESS: Metrology for a Sustainable Development, Rio de Janeiro, 17-22 September 2006.

[27] Fong, W.T., Ong, S.K. and Nee, A.Y.C. (2008) Methods for In-Field User Calibration of an Inertial Measurement Unit without External Equipment. Measurement Science \& Technology, 19, Article ID: 085202.

[28] Gebre-Egziabher, D., Elkaim, G.H., Powell, J.D. and Parkinson, B.W. (2001) A Non-Linear, Two-Step Estimation Algorithm for Calibrating Solid-State Srapdown Magnetometers. Proceedings of the International Conference on Integrated Navigation Systems, St-Petersburg, 28-30 May 2001.

[29] Foster, C.C. and Elkaim, G.H. (2008) Extension of a Two-Step Calibration Methodology to Include Nonorthogonal Sensor Axes. IEEE Transactions on Aerospace and Electronic Systems, 44, 1070-1078. https://doi.org/10.1109/TAES.2008.4655364

[30] Chiang, K.-W., Duong, T. and Liao, J.-K. (2013) The Performance Analysis of a Real-Time Integrated INS/GPS Vehicle Navigation System with Abnormal GPS Measurement Elimination. Sensors, 13, 10599. https://doi.org/10.3390/s130810599

[31] Farrell, J. (2008) Aided Navigation: GPS with High Rate Sensors. McGraw-Hill, New York.

[32] Salychev, O.S. (1998) Inertial Systems in Navigation and Geophysics. Bauman MSTU Press, Moscow.

Submit or recommend next manuscript to SCIRP and we will provide best service for you:

Accepting pre-submission inquiries through Email, Facebook, LinkedIn, Twitter, etc. A wide selection of journals (inclusive of 9 subjects, more than 200 journals) Providing 24-hour high-quality service User-friendly online submission system Fair and swift peer-review system Efficient typesetting and proofreading procedure Display of the result of downloads and visits, as well as the number of cited articles Maximum dissemination of your research work

Submit your manuscript at: http://papersubmission.scirp.org/

Or contact jec@scirp.org 